$\mathrm{MZ}-\mathrm{TH} / 10-31$

September 5, 2010

\title{
Updated Predictions for Higgs Production at the Tevatron and the LHC
}

\author{
Valentin Ahrens $^{a}$, Thomas Becher $^{b}$, Matthias Neubert $^{a}$, And Li Lin Yang $^{a}$ \\ ${ }^{a}$ Institut für Physik (THEP), Johannes Gutenberg-Universität \\ D-55099 Mainz, Germany \\ ${ }^{b}$ Institute for Theoretical Physics, University of Bern \\ CH-3012 Bern, Switzerland
}

\begin{abstract}
We present updated predictions for the total cross section for Higgs boson production through gluon fusion at hadron colliders. In addition to renormalization-group improvement at next-to-next-to-next-to-leading logarithmic accuracy, we incorporate the two-loop electroweak corrections, which leads to the most precise predictions at present. Numerical results are given for Higgs masses between $115 \mathrm{GeV}$ and $200 \mathrm{GeV}$ at the Tevatron with $\sqrt{s}=1.96 \mathrm{TeV}$ and the LHC with $\sqrt{s}=7-14 \mathrm{TeV}$.
\end{abstract}

The search for the Higgs boson is of the highest priority in the experimental programs at the Fermilab Tevatron and the CERN LHC. The lower bound for the Higgs mass obtained by the direct searches at the LEP, $m_{H} \geq 114.4 \mathrm{GeV}$ at the $95 \% \mathrm{CL}$, has been around for several years [1]. At the beginning of this year, the CDF and D0 Collaborations published a new result which excludes Higgs bosons with a mass around $2 m_{W}[2]$. After a recent update, the Tevatron exclusion now covers the range $158 \mathrm{GeV}<m_{H}<175 \mathrm{GeV}$ [3]. On the other hand, the electroweak precision measurements favor a relatively light Higgs boson with a mass well below $200 \mathrm{GeV}$ [4]. The LHC has started operation recently, and the standard model Higgs boson, if it exists, should be within reach in the next few years.

At hadron colliders, the most important production channel for the Higgs boson is the gluon fusion process. Much effort has been devoted to improving the theoretical predictions for this process, especially since it is well known that the total cross section suffers from huge QCD corrections [5-9]. In the recent papers [10,11], we have pointed out that a large portion of these corrections comes from enhanced contributions of the form $\left(C_{A} \pi \alpha_{s}\right)^{n}$, which arise in the analytic continuation of the gluon form factor from space-like to time-like momentum transfer. In those two papers, these large contributions, as well as threshold enhanced terms, were 
resummed to all orders in $\alpha_{s}$ at next-to-next-to-next-to-leading logarithmic $\left(\mathrm{N}^{3} \mathrm{LL}\right)$ accuracy using renormalization-group ( $\mathrm{RG}$ ) methods.

It is however necessary to update the numerical predictions presented in [11]. One reason is that there we have only provided results for the LHC at $\sqrt{s}=14 \mathrm{TeV}$, while it is now clear that the LHC will operate at a lower energy for two or more years. Another reason is the recent effort to evaluate the electroweak corrections to this process [12-15]. Given that QCD effects are well under control in our result (less than 3\% remaining scale uncertainty and perfect perturbative convergence), these electroweak corrections, which can be as large as $6 \%$, are non-negligible and should be included. The $\mathcal{O}(\alpha)$ electroweak corrections can be split into two parts. The part involving a light quark loop was computed in [12]. The part involving the top quark in the loop was first calculated in [13] as an expansion in $m_{H}^{2} /\left(4 m_{W}^{2}\right)$, which is therefore formally valid only for $m_{H}<2 m_{W}$. The complete $\mathcal{O}(\alpha)$ corrections including the exact top quark contributions were later evaluated in $[14,15]$ using numerical methods.

Given the $\mathcal{O}(\alpha)$ corrections, there are still ambiguities in how to combine them with the QCD corrections. In [14] two schemes were proposed, which were called the "partial factorization" scheme and the "complete factorization" scheme. In the partial factorization scheme the $\mathcal{O}(\alpha)$ corrections are simply added to the QCD corrected cross section, while in the complete factorization scheme the $\mathcal{O}(\alpha)$ corrections serve as a prefactor in front of the QCD corrected cross section, which then generate terms of $\mathcal{O}\left(\alpha \alpha_{s}^{n}\right)$. Since the QCD corrections in fixed-order perturbation theory are large, these two schemes can have non-negligible differences, and it was not known at that time which one is better without an explicit calculation of the $\mathcal{O}\left(\alpha \alpha_{s}\right)$ contributions. This task has been undertaken in [16], where it was demonstrated that although the complete factorization does not hold exactly, numerically it gives a good approximation to the $\mathcal{O}\left(\alpha \alpha_{s}\right)$ terms. We will therefore adopt the complete factorization approach in our result. The relative contribution of the electroweak corrections is about $4 \%$ for $m_{H} \sim 100 \mathrm{GeV}$, rises to about $6 \%$ at the $W W$ threshold, and quickly drops to about $-2 \%$ for $m_{H} \sim 200 \mathrm{GeV}$.

The uncertainties in our predictions come from several sources. The uncertainty concerning unknown higher-order QCD corrections can be estimated from the scale dependence of the cross section. In our approach there are four scales: $\mu_{t}, \mu_{h}, \mu_{s}$ and $\mu_{f}$, and we estimate the scale uncertainty by varying the scales up and down from their central values and adding in quadrature the associated variations of the cross section (see [11] for details). The resulting uncertainty is less than 3\% for both the Tevatron and the LHC. The uncertainty inherent in the experimental determinations of the parton distribution functions (PDFs) and the strong coupling constant $\alpha_{s}$ can be estimated at 90\% CL using modern PDF sets [17-19]. It is found to range from $11 \%$ to $15 \%$ for the Tevatron and is about $8 \%$ for the LHC. On top of these there is a small uncertainty coming from the use of the heavy top limit in the calculation of QCD corrections, which has been shown to be a very good approximation for a relatively light Higgs boson at NLO [20] and recently also at NNLO [21,22]. We also treat the perturbative correction to Higgs production via a $b$-quark loop in the heavy quark limit. This approximation is fairly crude and results in an additional uncertainty of about $1 \%$ in the total cross section.

In our numerical evaluation we take the input parameters as [23, 24]

$$
m_{t}=173.1 \mathrm{GeV}, \quad \bar{m}_{b}\left(\bar{m}_{b}\right)=4.2 \mathrm{GeV},
$$




\begin{tabular}{|c|c|c|c|c|}
\hline$\overline{m_{H}[\mathrm{GeV}]}$ & Tevatron & LHC (7 TeV) & LHC $(10 \mathrm{TeV})$ & $\overline{\mathrm{LHC}}(14 \mathrm{TeV})$ \\
\hline 115 & $1.215_{-0.007-0.135}^{+0.031+0.141}$ & $18.19_{-0.14-1.39}^{+0.53+1.46}$ & $33.7_{-0.2-2.5}^{+1.0+2.6}$ & $57.9_{-0.3-4.2}^{+1.6+4.4}$ \\
\hline 120 & $1.073_{-0.006-0.121}^{+0.026+0.126}$ & $16.73_{-0.13-1.28}^{+0.48+1.34}$ & $31.2_{-0.2-2.3}^{+0.9+2.4}$ & $54.0_{-0.3-3.9}^{+1.5+4.1}$ \\
\hline 125 & $0.950_{-0.005-0.108}^{+0.022+0.113}$ & $15.43_{-0.12-1.18}^{+0.44+1.23}$ & $29.0_{-0.2-2.1}^{+0.8+2.2}$ & $50.4_{-0.3-3.6}^{+1.4+3.8}$ \\
\hline 130 & $0.844_{-0.004-0.098}^{+0.019+0.102}$ & $14.27_{-0.11-1.09}^{+0.40+1.14}$ & $27.0_{-0.2-2.0}^{+0.7+2.1}$ & $47.2_{-0.3-3.4}^{+1.3+3.5}$ \\
\hline 135 & $0.753_{-0.004-0.088}^{+0.016+0.093}$ & $13.23_{-0.10-1.01}^{+0.36+1.06}$ & $25.2_{-0.2-1.8}^{+0.7+1.9}$ & $44.3_{-0.3-3.2}^{+1.2+3.3}$ \\
\hline 140 & $0.672_{-0.003-0.080}^{+0.014+0.084}$ & $12.29_{-0.09-0.94}^{+0.33+0.98}$ & $23.5_{-0.2-1.7}^{+0.6+1.8}$ & $41.6_{-0.3-3.0}^{+1.1+3.1}$ \\
\hline 145 & $0.602_{-0.003-0.072}^{+0.012+0.076}$ & $11.44_{-0.08-0.88}^{+0.31+0.91}$ & $22.1_{-0.1-1.6}^{+0.6+1.7}$ & $39.2_{-0.2-2.8}^{+1.0+2.9}$ \\
\hline 150 & $0.541_{-0.002-0.066}^{+0.010+0.070}$ & $10.67_{-0.08-0.82}^{+0.28+0.85}$ & $20.7_{-0.1-1.5}^{+0.5+1.6}$ & $37.0_{-0.2-2.6}^{+1.0+2.7}$ \\
\hline 155 & $0.486_{-0.002-0.060}^{+0.009+0.064}$ & $9.95_{-0.07-0.77}^{+0.26+0.80}$ & $19.4_{-0.1-1.4}^{+0.5+1.5}$ & $34.9_{-0.2-2.5}^{+0.9+2.6}$ \\
\hline 160 & $0.433_{-0.002-0.054}^{+0.008+0.058}$ & $9.21_{-0.07-0.71}^{+0.24+0.74}$ & $18.1_{-0.1-1.3}^{+0.5+1.4}$ & $32.7_{-0.2-2.3}^{+0.8+2.4}$ \\
\hline 165 & $0.385_{-0.002-0.049}^{+0.006+0.052}$ & $8.50_{-0.06-0.66}^{+0.22+0.68}$ & $16.8_{-0.1-1.2}^{+0.4+1.3}$ & $30.5_{-0.2-2.1}^{+0.8+2.2}$ \\
\hline 170 & $0.345_{-0.002-0.044}^{+0.005+0.047}$ & $7.89_{-0.06-0.61}^{+0.20+0.63}$ & $15.7_{-0.1-1.1}^{+0.4+1.2}$ & $28.6_{-0.2-2.0}^{+0.7+2.1}$ \\
\hline 175 & $0.310_{-0.001-0.040}^{+0.005+0.043}$ & $\begin{array}{l}7.36_{-0.05-0.57}^{+0.18+0.59} \\
\end{array}$ & $14.7_{-0.1-1.1}^{+0.4+1.1}$ & $27.0_{-0.2-1.9}^{+0.7+1.9}$ \\
\hline 180 & $0.280_{-0.001-0.037}^{+0.004+0.040}$ & $6.88_{-0.05-0.54}^{+0.17+0.56}$ & $13.8_{-0.1-1.0}^{+0.3+1.0}$ & $25.5_{-0.2-1.8}^{+0.6+1.8}$ \\
\hline 185 & $0.252_{-0.001-0.033}^{+0.003+0.036}$ & $6.42_{-0.04-0.50}^{+0.15+0.52}$ & $13.0_{-0.1-0.9}^{+0.3+1.0}$ & $24.0_{-0.1-1.7}^{+0.6+1.7}$ \\
\hline 190 & $0.228_{-0.001-0.031}^{+0.003+0.033}$ & $6.02_{-0.04-0.47}^{+0.14+0.49}$ & $12.2_{-0.1-0.9}^{+0.3+0.9}$ & $22.7_{-0.1-1.6}^{+0.5+1.6}$ \\
\hline 195 & $0.207_{-0.001-0.028}^{+0.002+0.031}$ & $5.67_{-0.04-0.45}^{+0.13+0.46}$ & $11.6_{-0.1-0.8}^{+0.3+0.9}$ & $21.6_{-0.1-1.5}^{+0.5+1.6}$ \\
\hline 200 & $0.189_{-0.001-0.026}^{+0.002+0.028}$ & $5.35_{-0.03-0.42}^{+0.12+0.44}$ & $11.0_{-0.1-0.8}^{+0.3+0.8}$ & $20.6_{-0.1-1.4}^{+0.5+1.5}$ \\
\hline
\end{tabular}

Table 1: Cross sections (in pb) for different Higgs masses at the Tevatron and the LHC, using MSTW2008NNLO PDFs. The first error accounts for scale variations, while the second one reflects the combined uncertainty from the PDFs and $\alpha_{s}$.

$$
m_{Z}=91.1876 \mathrm{GeV}, \quad G_{F}\left(m_{Z}\right)=1.16208 \cdot 10^{-5} \mathrm{GeV}^{-2},
$$

and by default use the MSTW2008NNLO PDFs [25] with $\alpha_{s}\left(m_{Z}\right)=0.11707$. The other electroweak parameters are the same as in [14]. For comparison, we also show numbers obtained using the CT10 and NNPDF2.0 PDFs $[26,27]$, with the corresponding values of $\alpha_{s}\left(m_{Z}\right)$. We note, however, that these are NLO PDFs and therefore less well suited for our calculation.

Our main results are summarized in Table 1, where our best predictions for the cross section at the Tevatron with $\sqrt{s}=1.96 \mathrm{TeV}$ and the LHC with $\sqrt{s}=7,10,14 \mathrm{TeV}$ using MSTW2008NNLO PDFs are shown. In Figure 1, we show the cross sections as functions of $m_{H}$, with bands representing the scale uncertainties. We have also depicted the LO and NLO RG-improved cross sections in Figure 1, to show the good perturbative convergence of our result. In Figure 2, we plot the central values of the cross sections at the LHC for $m_{H}=120$, 160 and $200 \mathrm{GeV}$ as functions of $\sqrt{s}$. For comparison, in Table 2 and 3 we also show the cross sections using CT10 and NNPDF2.0 PDFs. They agree with the results in Table 1 within errors. To make it simple to update our results in the future, we include a Fortran program 

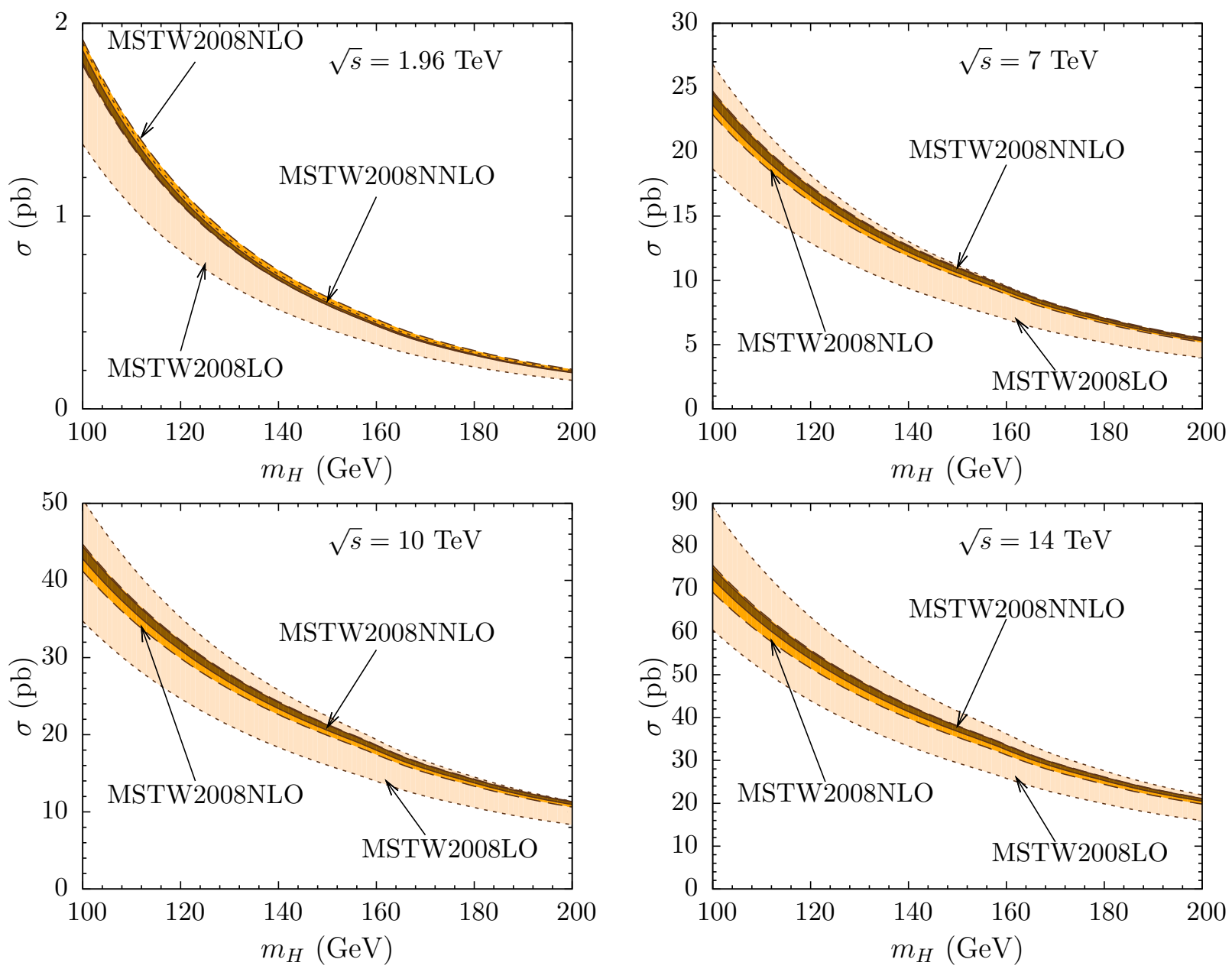

Figure 1: Cross sections at the Tevatron for $\sqrt{s}=1.96 \mathrm{TeV}$ and the LHC for $\sqrt{s}=7,10$, $14 \mathrm{TeV}$. Bands indicate scale uncertainties. Light, medium and dark bands represent LO (NLL), NLO (NNLL) and NNLO $\left(\mathrm{N}^{3} \mathrm{LL}\right)$ in RG-improved perturbation theory, respectively.

for download ${ }^{1}$.

In [28], the authors have also updated their predictions for Higgs production via gluon fusion combining soft gluon resummation and two-loop electroweak corrections. Our results differ in several important aspects from theirs:

- We work at $\mathrm{N}^{3} \mathrm{LL}$ accuracy rather than NNLL.

- We resum the enhanced contributions arising from the analytic continuation of the gluon form factor. This has been demonstrated to greatly improve the perturbative convergence.

- We work directly in momentum space rather than in Mellin moment space, which avoids the Landau pole ambiguity.

${ }^{1}$ http://projects.hepforge.org/rghiggs/ 


\begin{tabular}{|c|c|c|c|c|}
\hline$m_{H}[\mathrm{GeV}]$ & Tevatron & $\mathrm{LHC}(7 \mathrm{TeV})$ & LHC $(10 \mathrm{TeV})$ & LHC $(14 \mathrm{TeV})$ \\
\hline 115 & $1.215_{-0.007-0.095}^{+0.031+0.105}$ & $18.34_{-0.14-1.00}^{+0.54+0.95}$ & $34.1_{-0.2-1.9}^{+1.0+1.8}$ & $58.8_{-0.4-3.5}^{+1.7+3.1}$ \\
\hline 120 & $1.073_{-0.005-0.087}^{+0.026+0.096}$ & $16.86_{-0.13-0.91}^{+0.49+0.87}$ & $31.5_{-0.2-1.8}^{+0.9+1.6}$ & $54.7_{-0.3-3.2}^{+1.6+2.9}$ \\
\hline 125 & $0.950_{-0.005-0.079}^{+0.022+0.088}$ & $15.54_{-0.12-0.83}^{+0.45+0.80}$ & $29.3_{-0.2-1.6}^{+0.8+1.5}$ & $51.1_{-0.3-3.0}^{+1.4+2.6}$ \\
\hline 130 & $0.845_{-0.004-0.072}^{+0.019+0.081}$ & $14.36_{-0.11-0.76}^{+0.41+0.74}$ & $27.2_{-0.2-1.5}^{+0.8+1.4}$ & $47.8_{-0.3-2.7}^{+1.3+2.5}$ \\
\hline 135 & $0.753_{-0.004-0.067}^{+0.016+0.075}$ & $13.31_{-0.10-0.70}^{+0.37+0.68}$ & $25.4_{-0.2-1.4}^{+0.7+1.3}$ & $44.8_{-0.3-2.5}^{+1.2+2.3}$ \\
\hline 140 & $0.673_{-0.003-0.061}^{+0.014+0.069}$ & $12.35_{-0.09-0.65}^{+0.34+0.63}$ & $23.7_{-0.2-1.3}^{+0.7+1.2}$ & $42.1_{-0.3-2.3}^{+1.1+2.1}$ \\
\hline 145 & $0.604_{-0.003-0.057}^{+0.012+0.064}$ & $11.50_{-0.08-0.60}^{+0.31+0.59}$ & $22.2_{-0.2-1.2}^{+0.6+1.1}$ & $39.7_{-0.2-2.2}^{+1.1+2.0}$ \\
\hline 150 & $0.542_{-0.002-0.052}^{+0.010+0.059}$ & $10.71_{-0.08-0.56}^{+0.29+0.55}$ & $20.9_{-0.1-1.1}^{+0.6+1.0}$ & $37.4_{-0.2-2.0}^{+1.0+1.9}$ \\
\hline 155 & $0.487_{-0.002-0.049}^{+0.009+0.055}$ & $9.99_{-0.07-0.52}^{+0.26+0.51}$ & $19.6_{-0.1-1.0}^{+0.5+1.0}$ & $35.2_{-0.2-1.9}^{+0.9+1.7}$ \\
\hline 160 & $0.435_{-0.002-0.045}^{+0.008+0.050}$ & $9.24_{-0.07-0.48}^{+0.24+0.48}$ & $18.2_{-0.1-0.9}^{+0.5+0.9}$ & $33.0_{-0.2-1.7}^{+0.9+1.6}$ \\
\hline 165 & $0.387_{-0.002-0.041}^{+0.007+0.046}$ & $8.52_{-0.06-0.44}^{+0.22+0.44}$ & $16.9_{-0.1-0.9}^{+0.4+0.8}$ & $30.7_{-0.2-1.6}^{+0.8+1.5}$ \\
\hline 170 & $0.347_{-0.002-0.038}^{+0.006+0.043}$ & $7.91_{-0.05-0.41}^{+0.20+0.41}$ & $15.8_{-0.1-0.8}^{+0.4+0.8}$ & $28.8_{-0.2-1.5}^{+0.7+1.4}$ \\
\hline 175 & $0.313_{-0.001-0.035}^{+0.005+0.039}$ & $7.38_{-0.05-0.38}^{+0.19+0.38}$ & $14.8_{-0.1-0.7}^{+0.4+0.7}$ & $27.2_{-0.2-1.4}^{+0.7+1.3}$ \\
\hline 180 & $0.282_{-0.001-0.032}^{+0.004+0.037}$ & $6.89_{-0.05-0.36}^{+0.17+0.36}$ & $13.9_{-0.1-0.7}^{+0.3+0.7}$ & $25.7_{-0.2-1.3}^{+0.6+1.2}$ \\
\hline 185 & $0.254_{-0.001-0.030}^{+0.004+0.034}$ & $6.43_{-0.04-0.33}^{+0.16+0.34}$ & $13.1_{-0.1-0.7}^{+0.3+0.6}$ & $24.2_{-0.1-1.2}^{+0.6+1.1}$ \\
\hline 190 & $0.230_{-0.001-0.028}^{+0.003+0.032}$ & $6.02_{-0.04-0.31}^{+0.15+0.32}$ & $12.3_{-0.1-0.6}^{+0.3+0.6}$ & $22.9_{-0.1-1.2}^{+0.6+1.1}$ \\
\hline 195 & $0.210_{-0.001-0.026}^{+0.003+0.030}$ & $5.67_{-0.04-0.30}^{+0.14+0.30}$ & $11.6_{-0.1-0.6}^{+0.3+0.6}$ & $21.8_{-0.1-1.1}^{+0.5+1.0}$ \\
\hline 200 & $0.191_{-0.001-0.024}^{+0.002+0.028}$ & $5.35_{-0.03-0.28}^{+0.13+0.29}$ & $11.1_{-0.1-0.5}^{+0.3+0.5}$ & $20.8_{-0.1-1.0}^{+0.5+1.0}$ \\
\hline
\end{tabular}

Table 2: Cross sections (in pb) for different Higgs masses at the Tevatron and the LHC, using CT10 PDFs with $\alpha_{s}\left(m_{Z}\right)=0.118$.

Therefore, we believe that our results are the most precise predictions for the total Higgs production cross sections to date. With the higher-order perturbative corrections under control, the main uncertainties now arise from the experimental determinations of the PDFs and $\alpha_{s}$.

\section{Acknowledgments}

We are grateful to Alessandro Vicini, Roberto Bonciani and Juan Rojo for useful discussions. We thank Stefano Actis for pointing us to the grid files for the electroweak corrections.

\section{References}

[1] R. Barate et al. [ALEPH, DELPHI, L3 and OPAL Collaborations and the LEP Working Group for Higgs boson searches], Phys. Lett. B 565, 61 (2003) [arXiv:hep-ex/0306033].

[2] T. Aaltonen et al. [CDF and D0 Collaborations], Phys. Rev. Lett. 104, 061802 (2010) [arXiv:1001.4162 [hep-ex]]. 


\begin{tabular}{|c|c|c|c|c|}
\hline$m_{H}[\mathrm{GeV}]$ & Tevatron & $\mathrm{LHC}(7 \mathrm{TeV})$ & LHC $(10 \mathrm{TeV})$ & LHC $(14 \mathrm{TeV})$ \\
\hline 115 & $1.341_{-0.018-0.143}^{+0.037+0.143}$ & $19.35_{-0.29-1.36}^{+0.60+1.36}$ & $35.4_{-0.5-2.4}^{+1.1+2.4}$ & $60.3_{-0.7-3.9}^{+1.8+3.9}$ \\
\hline 120 & $1.184_{-0.016-0.129}^{+0.032+0.129}$ & $17.82_{-0.29-1.25}^{+0.54+1.25}$ & $32.8_{-0.5-2.2}^{+1.0+2.2}$ & $56.3_{-0.7-3.7}^{+1.7+3.7}$ \\
\hline 125 & $1.049_{-0.014-0.116}^{+0.027+0.116}$ & $16.45_{-0.28-1.15}^{+0.50+1.15}$ & $30.5_{-0.5-2.0}^{+0.9+2.0}$ & $52.6_{-0.8-3.4}^{+1.5+3.4}$ \\
\hline 130 & $0.932_{-0.013-0.105}^{+0.023+0.105}$ & $15.23_{-0.28-1.07}^{+0.45+1.07}$ & $28.5_{-0.5-1.9}^{+0.8+1.9}$ & $49.3_{-0.8-3.2}^{+1.4+3.2}$ \\
\hline 135 & $0.831_{-0.011-0.096}^{+0.020+0.096}$ & $14.13_{-0.27-0.99}^{+0.41+0.99}$ & $26.6_{-0.5-1.8}^{+0.8+1.8}$ & $46.3_{-0.8-3.0}^{+1.3+3.0}$ \\
\hline 140 & $0.742_{-0.010-0.087}^{+0.017+0.087}$ & $13.14_{-0.26-0.93}^{+0.38+0.93}$ & $24.9_{-0.5-1.7}^{+0.7+1.7}$ & $43.6_{-0.8-2.8}^{+1.2+2.8}$ \\
\hline 145 & $0.665_{-0.009-0.080}^{+0.015+0.080}$ & $12.24_{-0.25-0.86}^{+0.35+0.86}$ & $23.3_{-0.5-1.5}^{+0.7+1.5}$ & $41.1_{-0.8-2.6}^{+1.1+2.6}$ \\
\hline 150 & $0.597_{-0.008-0.073}^{+0.013+0.073}$ & $11.42_{-0.24-0.81}^{+0.32+0.81}$ & $21.9_{-0.4-1.5}^{+0.6+1.5}$ & $38.8_{-0.7-2.5}^{+1.1+2.5}$ \\
\hline 155 & $0.536_{-0.007-0.067}^{+0.011+0.067}$ & $10.66_{-0.23-0.76}^{+0.30+0.76}$ & $20.6_{-0.4-1.4}^{+0.6+1.4}$ & $36.6_{-0.7-2.3}^{+1.0+2.3}$ \\
\hline 160 & $0.478_{-0.006-0.061}^{+0.010+0.061}$ & $9.88_{-0.22-0.70}^{+0.27+0.70}$ & $19.2_{-0.4-1.3}^{+0.5+1.3}$ & $34.3_{-0.7-2.2}^{+0.9+2.2}$ \\
\hline 165 & $0.425_{-0.005-0.055}^{+0.008+0.055}$ & $9.11_{-0.21-0.65}^{+0.25+0.65}$ & $17.8_{-0.4-1.2}^{+0.5+1.2}$ & $32.0_{-0.7-2.0}^{+0.9+2.0}$ \\
\hline 170 & $0.380_{-0.005-0.050}^{+0.007+0.050}$ & $8.46_{-0.19-0.61}^{+0.24+0.61}$ & $16.6_{-0.4-1.1}^{+0.5+1.1}$ & $30.0_{-0.6-1.9}^{+0.8+1.9}$ \\
\hline 175 & $0.342_{-0.004-0.046}^{+0.006+0.046}$ & $7.90_{-0.18-0.57}^{+0.22+0.57}$ & $15.6_{-0.4-1.0}^{+0.4+1.0}$ & $28.4_{-0.6-1.8}^{+0.8+1.8}$ \\
\hline 180 & $0.308_{-0.003-0.042}^{+0.005+0.042}$ & $\begin{array}{l}7.38_{-0.17-0.53}^{+0.20+0.53} \\
\end{array}$ & $14.7_{-0.3-1.0}^{+0.4+1.0}$ & $26.8_{-0.6-1.7}^{+0.7+1.7}$ \\
\hline 185 & $0.277_{-0.003-0.039}^{+0.005+0.039}$ & $6.90_{-0.16-0.50}^{+0.19+0.50}$ & $13.8_{-0.3-0.9}^{+0.4+0.9}$ & $25.3_{-0.6-1.6}^{+0.7+1.6}$ \\
\hline 190 & $0.250_{-0.002-0.036}^{+0.004+0.036}$ & $6.46_{-0.15-0.47}^{+0.18+0.47}$ & $13.0_{-0.3-0.9}^{+0.4+0.9}$ & $23.9_{-0.5-1.5}^{+0.7+1.5}$ \\
\hline 195 & $0.227_{-0.002-0.033}^{+0.004+0.033}$ & $6.08_{-0.14-0.44}^{+0.17+0.44}$ & $12.3_{-0.3-0.8}^{+0.4+0.8}$ & $22.8_{-0.5-1.4}^{+0.6+1.4}$ \\
\hline 200 & $0.207_{-0.002-0.031}^{+0.003+0.031}$ & $5.74_{-0.13-0.42}^{+0.17+0.42}$ & $11.7_{-0.3-0.8}^{+0.3+0.8}$ & $21.7_{-0.5-1.4}^{+0.6+1.4}$ \\
\hline
\end{tabular}

Table 3: Cross sections (in pb) for different Higgs masses at the Tevatron and the LHC, using NNPDF2.0 PDFs with $\alpha_{s}\left(m_{Z}\right)=0.119$.

[3] [The TEVNPH Working Group of the CDF and D0 Collaborations], arXiv:1007.4587 [hep-ex].

[4] J. Alcaraz [The ALEPH, CDF, D0, DELPHI, L3, OPAL, SLD Collaborations, the LEP Electroweak Working Group, the Tevatron Electroweak Working Group, and the SLD electroweak and heavy flavour groups], arXiv:0911.2604 [hep-ex].

[5] S. Dawson, Nucl. Phys. B 359, 283 (1991).

[6] A. Djouadi, M. Spira and P. M. Zerwas, Phys. Lett. B 264, 440 (1991).

[7] R. V. Harlander and W. B. Kilgore, Phys. Rev. Lett. 88, 201801 (2002) [arXiv:hep$\mathrm{ph} / 0201206]$.

[8] C. Anastasiou and K. Melnikov, Nucl. Phys. B 646, 220 (2002) [arXiv:hep-ph/0207004].

[9] V. Ravindran, J. Smith and W. L. van Neerven, Nucl. Phys. B 665, 325 (2003) [arXiv:hep-ph/0302135]. 


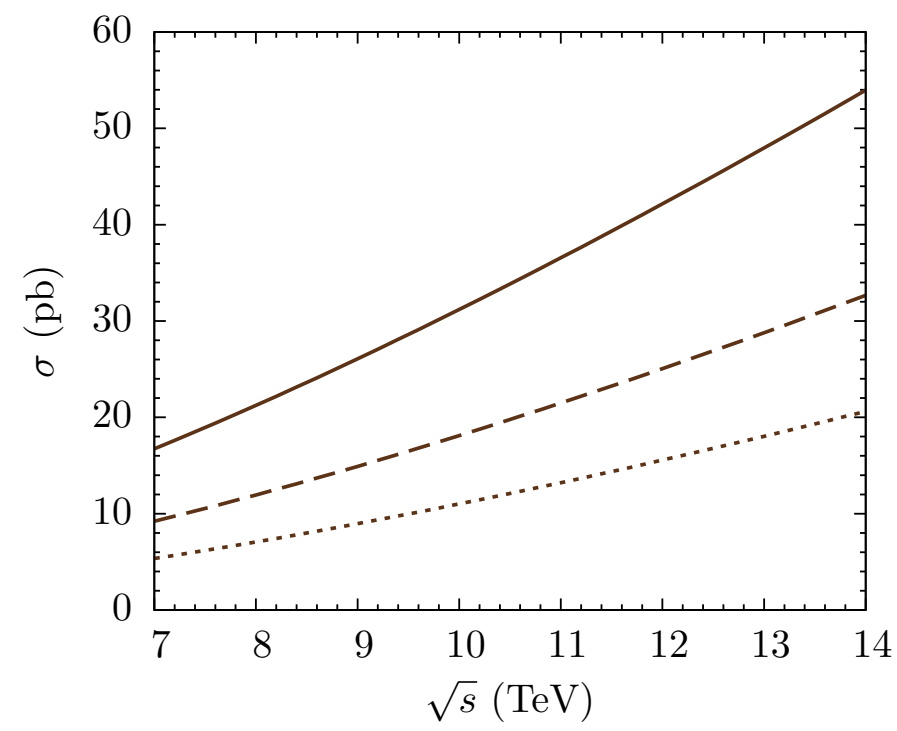

Figure 2: Cross sections as functions of the center-of-mass energy at the LHC for Higgs masses being $120 \mathrm{GeV}$ (solid), $160 \mathrm{GeV}$ (dashed) and $200 \mathrm{GeV}$ (dotted).

[10] V. Ahrens, T. Becher, M. Neubert and L. L. Yang, Phys. Rev. D 79, 033013 (2009) [arXiv:0808.3008 [hep-ph]].

[11] V. Ahrens, T. Becher, M. Neubert and L. L. Yang, Eur. Phys. J. C 62, 333 (2009) [arXiv:0809.4283 [hep-ph]].

[12] U. Aglietti, R. Bonciani, G. Degrassi and A. Vicini, Phys. Lett. B 595, 432 (2004) [arXiv:hep-ph/0404071].

[13] G. Degrassi and F. Maltoni, Phys. Lett. B 600, 255 (2004) [arXiv:hep-ph/0407249].

[14] S. Actis, G. Passarino, C. Sturm and S. Uccirati, Phys. Lett. B 670, 12 (2008) [arXiv:0809.1301 [hep-ph]].

[15] S. Actis, G. Passarino, C. Sturm and S. Uccirati, Nucl. Phys. B 811, 182 (2009) [arXiv:0809.3667 [hep-ph]].

[16] C. Anastasiou, R. Boughezal and F. Petriello, JHEP 0904, 003 (2009) [arXiv:0811.3458 [hep-ph]].

[17] A. D. Martin, W. J. Stirling, R. S. Thorne and G. Watt, Eur. Phys. J. C 64, 653 (2009) [arXiv:0905.3531 [hep-ph]].

[18] H. L. Lai, J. Huston, Z. Li, P. Nadolsky, J. Pumplin, D. Stump and C. P. Yuan, arXiv:1004.4624 [hep-ph].

[19] F. Demartin, S. Forte, E. Mariani, J. Rojo and A. Vicini, Phys. Rev. D 82, 014002 (2010) [arXiv:1004.0962 [hep-ph]]. 
[20] M. Spira, A. Djouadi, D. Graudenz and P. M. Zerwas, Nucl. Phys. B 453, 17 (1995) [arXiv:hep-ph/9504378].

[21] R. V. Harlander and K. J. Ozeren, JHEP 0911, 088 (2009) [arXiv:0909.3420 [hep-ph]].

[22] A. Pak, M. Rogal and M. Steinhauser, JHEP 1002, 025 (2010) [arXiv:0911.4662 [hep$\mathrm{ph}]$.

[23] C. Amsler et al. [Particle Data Group], Phys. Lett. B 667, 1 (2008).

[24] Tevatron Electroweak Working Group and the CDF and D0 Collaborations, arXiv:0903.2503 [hep-ex].

[25] A. D. Martin, W. J. Stirling, R. S. Thorne and G. Watt, Eur. Phys. J. C 63, 189 (2009) [arXiv:0901.0002 [hep-ph]].

[26] H. L. Lai, M. Guzzi, J. Huston, Z. Li, P. M. Nadolsky, J. Pumplin and C. P. Yuan, arXiv:1007.2241 [hep-ph].

[27] R. D. Ball, L. Del Debbio, S. Forte, A. Guffanti, J. I. Latorre, J. Rojo and M. Ubiali, Nucl. Phys. B 838, 136 (2010) [arXiv:1002.4407 [hep-ph]].

[28] D. de Florian and M. Grazzini, Phys. Lett. B 674, 291 (2009) [arXiv:0901.2427 [hep-ph]]. 